\title{
7.3 Erkrankungen des Bewegungsapparates
}

\section{Stephan Reichenbach}

Die Erkrankungen des Bewegungsapparates, d.h. die Krankheiten der Gelenke, Knochen und Muskeln, verursachen weltweit am häufigsten Gesundheitsprobleme. Die dabei auftretenden Beschwerden reichen von leichten, vorübergehenden Beeinträchtigungen bis hin zu schweren, chronischen Behinderungen, welche schließlich zur Berentung führen können. Nur selten sind sie lebensbedrohlich sie schränken aber den Aktionsradius und damit die Lebensqualität der Betroffenen oft massiv ein. Dies führt zu großen sozioökonomischen Belastungen, nicht nur durch kostenintensive Therapien und Betreuungsangebote, sondern auch als Folge der verminderten Produktivität der Betroffenen.

In diesem Abschnitt betrachten wir die epidemiologische Bedeutung der wichtigsten Krankheitsbilder im muskuloskeletalen Bereich. Wir schauen auf die jeweiligen Risikofaktoren und erörtern, welche präventiven Maßnahmen viele dieser Krankheitsfälle verhindern könnten.

Schweizerische Lernziele: $\mathrm{CPH} 40$

$\mathrm{Zu}$ den klinisch und epidemiologisch relevanten Krankheitsbildern in dieser Gruppe gehören der Rückenschmerz (ICD-10, M40-M54), die Arthrose (ICD-10, M15-M19), die Osteoporose (ICD-10, M80-M85) sowie die rheumatoide Arthritis (ICD-10, M05-M14). Kardinalsymptome bei all diesen Erkrankungen sind Schmerzen, Bewegungseinschränkungen und damit einher gehender Funktionsverlust. Trotz der großen volkswirtschaftlichen Bedeutung dieser Krankheitsgruppe wurden bislang noch keine Interventionsstudien zur Primärprävention durchgeführt. Die hier vorgeschlagenen Präventionsmaßnahmen zielen primär auf die Verhinderung und Reduktion der bekannten und beeinflussbaren Risikofaktoren.

\subsubsection{Rückenschmerzen}

Unter dem Begriff Rückenschmerzen fasst man unabhängig von der Ursache alle Schmerzzustände im Bereich des Rückens zusammen. In ca. 20\% der Fälle sind dies klar umschriebene Krankheitsbilder. Meist ist jedoch eine genaue Zuordnung zu einem definierten Krankheitsbild nicht möglich, sodass die Schmerzen dann als unspezifisch klassifiziert werden (ca. 80\% der Fälle). Die unspezifischen Rückenschmerzen werden nach ihrer zeitlichen Dauer in akute $(<$ als 1 Monat), subakute $(<$ als 3 Monate) und chronische Schmerzzustände eingeteilt. Eine Chronifizierung der Schmerzen tritt in etwa $10 \%$ der Fälle ein (s. Box 7.3.1). 
Box 7.3.1: „Back pain - don't take it lying down“ - Massenmedienkampagne in Australien (1997-1999)

Gelegentliche Rückenschmerzen sind häufig, dauern meist nur kurze Zeit an, und gehören praktisch zu unserem Leben dazu. Entgegen dem subjektiven Empfinden sind sie in den letzten 25 Jahren nicht häufiger geworden. Wir fühlen uns allerdings durch Rückenschmerzen heute mehr beeinträchtigt als früher und nehmen deswegen auch öfter therapeutische Hilfe in Anspruch. Meist sind dann subjektives Empfinden, radiologische Befunde und Störungen der Funktionsfähigkeit nicht miteinander in Einklang zu bringen. Bei bis zu 90\% der Betroffenen gehen die Beschwerden auch ohne spezielle Behandlung innerhalb von sechs Wochen zurück.

Im australischen Bundesstaat Viktoria wurde deshalb zwischen 1997 und 1999 eine Aufklärungskampagne im Fernsehen durchgeführt (Videoclips hierzu auf unserer Lehrbuch-Homepage). Prominente aus Sport, Kultur und Gesundheitswesen erläuterten den Zuschauern anhand einfacher Regeln sinnvolles Verhalten beim Auftreten von Rückenschmerzen:

- Rückenschmerzen sind in der Regel zwar lästig, aber harmlos.

- Die dadurch hervorgerufenen Einschränkungen können durch eine positive Grundhaltung reduziert werden.

- Eine spezifische Behandlung ist meist nicht nötig. Keine Bettruhe, Gymnastik und keine Krankschreibung, stattdessen Weiterführung der bisherigen Tätigkeiten.

Eine anschließende Studie konnte nachweisen, dass sich dadurch die Bewertung des Rückenschmerzes in der Bevölkerung änderte. Es kam zu einem signifikanten Rückgang der Behandlungskosten, der Krankheitstage und der Sozialversicherungskosten.

\section{Epidemiologische Daten}

- Burden of Disease: Hierzu fehlen derzeit noch genaue Zahlen. Eine WHO-Studie schätzte die Krankheitslast durch Rückenschmerzen im Jahr 2004 auf 2,5 Millionen DALYs, was 0,09\% der weltweiten Burden of Disease entspricht.

- Mortalität: Es gibt keine Hinweise darauf, dass der unspezifische Rückenschmerz mit einer erhöhten Mortalität einhergeht.

- Inzidenz: Aufgrund der oft unklaren Zuordnung und des möglichen episodischen Verlaufs mit Rezidiven sind Angaben zur Inzidenz schwierig. In den meisten HighIncome-Ländern liegt die jährliche Neuerkrankungsrate bei 4.000-5.000 pro 100.000 Einwohner.

- Prävalenz: Die Lebenszeitprävalenz variiert in den High-income Ländern zwischen $60 \%$ und $85 \%$, d. h. die meisten Menschen leiden im Laufe ihres Lebens mindestens einmal an Rückenschmerzen. In „Entwicklungsländern“ liegt die Lebenszeitprävalenz mit durchschnittlich 60.000 pro 100.000 Einwohner etwas niedriger. 


\section{Risikofaktoren}

Zu den mechanischen Risikofaktoren gehören das schwere Heben von Lasten am Arbeitsplatz, eine kauernde, gebeugte oder gedrehte Körperhaltungen sowie die Exposition gegenüber Vibrationen (s. Kap. 6.3.2). Ebenso wichtig sind psychosoziale Begleitfaktoren wie Unzufriedenheit am Arbeitsplatz, monotone Arbeit, Depressivität und Somatisierung. Dies gilt insbesondere im Hinblick auf eine mögliche Chronifizierung.

\section{Prävention}

- Maßnahmen der Verhaltensprävention sind muskelkräftigende sowie die Ausdauer fördernde sportliche Aktivitäten.

- Zu den Maßnahmen der Verhältnisprävention gehört es, an Arbeitsplätzen monotone Arbeitsabläufe und ein belastendes Arbeitsklima zu vermeiden. Dort wo schweres Heben unumgänglich ist, wie z.B. in der Krankenpflege, auf dem Bau oder in der Landwirtschaft, sollte eine ergonomische Schulung der Arbeitskräfte vorgenommen werden (s.a. Kap. 6.3.2).

\subsubsection{Arthrose}

Unter dem Krankheitsbild der Arthrose versteht man ein progressives Gelenkversagen aufgrund eines chronischen Ab- und Umbauprozesses, welcher alle Strukturen eines Gelenkes (Knorpel, subchondraler Knochen, Synovia, Sehnen und Muskeln) betreffen kann. Man unterscheidet dabei die primäre Arthrose, die ohne erkennbare Ursache entstanden ist, von der sekundären Arthrose, die z.B. auf ein Trauma zurückgeführt werden kann.

\section{Epidemiologische Daten}

- Burden of Disease: Aufgrund der recht hohen Einschränkung der Lebensqualität durch die Erkrankung wird die in DALYs ausgedrückte Krankheitslast der Arthrose mit durchschnittlich 200 bis 400 verlorenen, gesunden Lebensjahren pro 100.000 Einwohner angegeben. Sie ist damit mehr als doppelt so hoch wie bei der rheumatoiden Arthritis.

- Mortalität: Da die Arthrose nur in den seltensten Fällen das Leben der Betroffenen bedroht, liegen die Mortalitätsraten weltweit nur bei 0,0 bis 0,8 Todesfällen pro 100.000 Einwohner pro Jahr.

- Inzidenz: Die größte Zahl an diagnostizierten Neuerkrankungen findet man zwischen dem 65. und 75. Lebensjahr. Frauen sind häufiger betroffen als Männer. Modellrechnungen haben für die westlichen Industrienationen bei den Frauen eine jährliche Inzidenzrate von 1.350 und bei den Männern von 900 pro 100.000 Einwohner und Jahr ergeben.

- Prävalenz: Hier wird meist zwischen einer radiologisch nachgewiesenen und einer symptomatischen Arthrose unterschieden. Etwa 50\% der 65-Jährigen und $80 \%$ der 75-Jährigen zeigen bei uns radiologische Zeichen einer Arthrose. Am häufigsten 
betroffen sind dabei die Knie-, Hüft- und Fingergelenke. Die Prävalenz von Gelenkschmerzen steigt mit dem radiologischen Schweregrad der Arthrose an, wobei die Assoziation zwischen Röntgenbefund und Schmerzinzidenz jedoch nur mäßig ist. - Unterschiede in der Prävalenz der Arthrose existieren sowohl zwischen den einzelnen Regionen dieser Erde als auch in Bezug auf die bevorzugt betroffenen Gelenke. Die Hüftgelenksarthrose kommt z. B. in Südostasien deutlich seltener vor als in Europa und den USA. In den High-Income-Ländern ist die symptomatische Arthrose eher ein Problem der Schichten mit niedrigem sozioökonomischem Status. Hier besteht eine enge Korrelation zur Adipositas, welche ein Risikofaktor für die Entstehung der Erkrankung ist (s. Kap. 7.4).

\section{Risikofaktoren}

Zu den wichtigsten Risikofaktoren der Arthrose gehören Alter, Geschlecht, eine familiäre Prädisposition sowie Übergewicht. Aber auch Traumata, die einmalig oder wiederholt auf ein Gelenk einwirken (z. B. eine Meniskusoperation am Knie oder die berufsbedingte Überlastung der Gelenke in der Landwirtschaft), stellen Risikofaktoren dar. Diese können sich je nach Gelenk unterschiedlich stark auswirken. So spielt das Übergewicht bei der Entstehung der Kniearthrose eine wichtigere Rolle als bei der Entstehung der Hüftarthrose. Für das Fortschreiten der Arthrose scheint das Übergewicht dann interessanterweise jedoch kaum noch eine Bedeutung zu haben (Obesity paradoxon).

\section{Prävention}

- Die wichtigsten Maßnahmen der Verhaltensprävention sind das Vermeiden von Übergewicht sowie regelmäßige sportliche Aktivitäten, die die Muskulatur kräftigen und die Ausdauer fördern.

- Zu den Maßnahmen der Verhältnisprävention gehört z. B. die ergonomische Schulung von Personen an ihren Arbeitsplätzen.

\subsubsection{Osteoporose}

Die Osteoporose ist eine systemische Knochenerkrankung, charakterisiert durch eine niedrige Knochenmasse und eine beeinträchtigte Mikroarchitektur, die zu einer erhöhten Knochenbrüchigkeit und damit zu einem höheren Frakturrisiko führt. Typischerweise kommt es infolge einer Osteoporose zu Frakturen im Bereich der Wirbelkörper, des Oberschenkelhalses, des proximalen Humerus ${ }^{22}$ und des distalen ${ }^{23}$ Unterarms. Schmerzen durch osteoporotische Veränderungen treten besonders im Rückenbereich auf.

\section{Epidemiologische Daten}

- Burden of Disease: Hierzu gibt es bislang keine Zahlen. Es ist jedoch anzunehmen, dass die durch eine Osteoporose hervorgerufene Krankheitslast, ausgedrückt in

\footnotetext{
22 proximal: zum Rumpf in gelegen; Humerus: Oberarmknochen

${ }^{23}$ distal: körperfern, vom Rumpf weg liegend
} 
DALYs, beträchtlich ist, da $20 \%$ aller Personen mit Hüftfrakturen und $2 \%$ derjenigen mit einer Wirbelkörperfraktur im Anschluss an das Ereigniss in einem Pflegeheim weiterbetreut werden müssen.

- Mortalität: 10-20\% aller Patienten mit einer Oberschenkelhalsfraktur sterben innerhalb eines Jahres. Auch für Patienten mit einer Wirbelkörperfraktur wird eine zweibis dreifach erhöhte Mortalitätsrate gegenüber der Normalbevölkerung angegeben. Ähnliches gilt auch für andere Frakturen infolge Osteoporose.

- Inzidenz: Die Inzidenz der Osteoporose wird indirekt aufgrund der Häufigkeit der auftretenden Schenkelhalsfrakturen geschätzt, da diese in den High-income-Ländern zur Hospitalisation führen. Grundsätzlich lässt sich sagen, dass Frauen häufiger betroffen sind als Männer. Die höchsten Inzidenzraten werden in Nordeuropa und Nordamerika angegeben. In den USA liegt die Inzidenz in der Altersgruppe der 75-79-Jährigen beispielsweise bei den Männern bei 534, bei den Frauen bei 861 pro 100.000 Einwohner und Jahr. Die Zahlenangaben aus Afrika sind mit 2 pro 100.000 Einwohner und Jahr wesentlich niedriger. Da in den „Entwicklungsländern“ jedoch nicht alle Patienten mit einer Schenkelhalsfraktur hospitalisiert werden, ist ein Vergleich hier schwierig.

- Prävalenz: Die Prävalenz der Osteoporose wird indirekt aufgrund einer verminderten Knochendichte ermittelt, da das Risiko, eine Fraktur zu erleiden, mit der Verminderung der Knochendichte zunimmt. Die Krankheitshäufigkeit steigt in den westlichen Industrienationen mit dem Alter deutlich an, und zwar von $5 \%$ bei den 50 -jährigen Frauen bzw. 2,4\% bei den 50-jährigen Männern auf $50 \%$ bei den 85 -jährigen Frauen und 20\% bei den 85-jährigen Männern.

\section{Risikofaktoren}

Zu den Risikofaktoren der Osteoporose gehören neben Geschlecht und Alter auch eine frühe Menopause, ein niedriger Body-Mass-Index (BMI), Immobilität, Rauchen, Alkohol und verschiedene Medikamente (v. a. Glukokortikoide). Zusätzliche Faktoren, die das Risiko für eine Schenkelhalsfraktur erhöhen können, sind vorausgegangene Frakturen bei niedriger Knochendichte, Seh- und Gehstörungen, schlecht eingerichtete Wohnungen mit Stolperfallen sowie die Einnahme von Medikamenten, die Bewusstsein und Aufmerksamkeit beeinträchtigen können (z. B. Benzodiazepine).

\section{Prävention}

- Zu den Maßnahmen der Verhaltensprävention gehören neben dem Verzicht auf Nikotin und Alkohol vor allem das Vermeiden eines zu niedrigen Körpergewichts und einer lang andauernden Immobilität (von großer Bedeutung ist hier die frühzeitig einsetzende Physiotherapie).

- Die wichtigste verhältnispräventive Maßnahme besteht in der Verhinderung von Stolperstürzen durch das Vermeiden von Stolperfallen wie rutschenden Teppichen, Türschwellen etc. 


\subsubsection{Rheumatoide Arthritis}

Die rheumatoide Arthritis ist eine systemische Autoimmunerkrankung mit symmetrischem Befall von Gelenken, Sehnenscheiden und Schleimbeuteln. Krankheitstypisch sind Rheumaknoten und der Nachweis von Autoantikörpern.

\section{Epidemiologische Daten}

- Burden of Disease: Laut WHO beträgt die Krankheitslast durch die rheumatoide Arthritis in den OECD-Staaten etwas mehr als 80 verlorene, gesunde Lebensjahre pro 100.000 Einwohner.

- Mortalität: Patienten mit rheumatoider Arthritis weisen gegenüber der Normalbevölkerung eine mehr als doppelt so hohe Sterblichkeit auf. Die Mortalitätsraten variieren weltweit zwischen 0,1 und 2,7 Todesfällen pro 100.000 Einwohner pro Jahr.

- Inzidenz: Zahlen zur Inzidenz existieren nur für die High-income Länder, wo die Neuerkrankungsrate mit ca. 20-300 pro 100.000 Einwohner pro Jahr angegeben wird. Es gibt derzeit Hinweise darauf, dass die Inzidenz insbesondere bei den Frauen leicht abnimmt.

- Prävalenz: In den High-income-Ländern leiden heute zwischen 300 und 1.000 Menschen je 100.000 Einwohner an rheumatoider Arthritis. Für Entwicklungsländer gibt es Schätzungen, die von ca. 300 Krankheitsfällen je 100.000 Einwohner ausgehen. In Afrika wurden nur wenige Fälle beschrieben.

\section{Risikofaktoren}

Weibliches Geschlecht, eine familiäre Prädisposition sowie bestimmte genetische Faktoren (HLA DRB 1) erhöhen das Risiko, an rheumatoider Arthritis zu erkranken. Allerdings tritt die Erkrankung auch in der mediterranen Bevölkerung auf, wo das genannte Allel kaum vorkommt.

\section{Prävention}

- Verhaltensprävention: Bislang existieren keine Ansätze zur Primärprävention. Ziel der medikamentösen Behandlung mit so genannten Basismedikamenten muss es sein, Schäden an der Struktur der Gelenke zu verhindern (Tertiärprävention; s. Kap. 1).

- Verhältnisprävention: Bei der Arbeitsplatzgestaltung spielen ergotherapeutische Maßnahmen eine entscheidende Rolle. Auch die Abgabe von Hilfsmitteln erfolgt nach ergotherapeutischen Gesichtspunkten.

\section{Internet-Ressourcen}

Auf unserer Lehrbuch-Homepage (www.public-health-kompakt.de) finden Sie Hinweise auf weiterführende Literatur zusätzliche Tabellen, Videoclips sowie Links zu themenrelevanten Studien und Institutionen. 